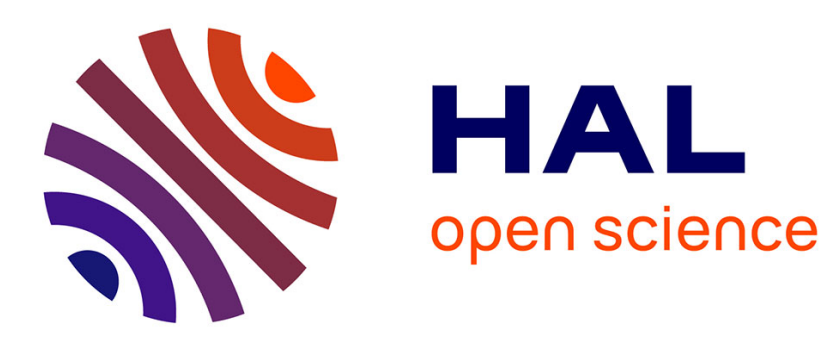

\title{
Geographic parthenogenesis in a consumer-resource model for sexual reproduction
}

Yixian Song, Stefan Scheu, Barbara Drossel

\section{To cite this version:}

Yixian Song, Stefan Scheu, Barbara Drossel. Geographic parthenogenesis in a consumerresource model for sexual reproduction. Journal of Theoretical Biology, 2011, 273 (1), pp.55. 10.1016/j.jtbi.2010.12.020 . hal-00669202

\section{HAL Id: hal-00669202 https://hal.science/hal-00669202}

Submitted on 12 Feb 2012

HAL is a multi-disciplinary open access archive for the deposit and dissemination of scientific research documents, whether they are published or not. The documents may come from teaching and research institutions in France or abroad, or from public or private research centers.
L'archive ouverte pluridisciplinaire HAL, est destinée au dépôt et à la diffusion de documents scientifiques de niveau recherche, publiés ou non, émanant des établissements d'enseignement et de recherche français ou étrangers, des laboratoires publics ou privés. 


\section{Author's Accepted Manuscript}

Geographic parthenogenesis in a consumer-resource model for sexual reproduction

Yixian Song, Stefan Scheu, Barbara Drossel

PII: S0022-5193(10)00675-2

DOI: doi:10.1016/j.jtbi.2010.12.020

Reference: YJTBI 6289

To appear in: $\quad$ Journal of Theoretical Biology

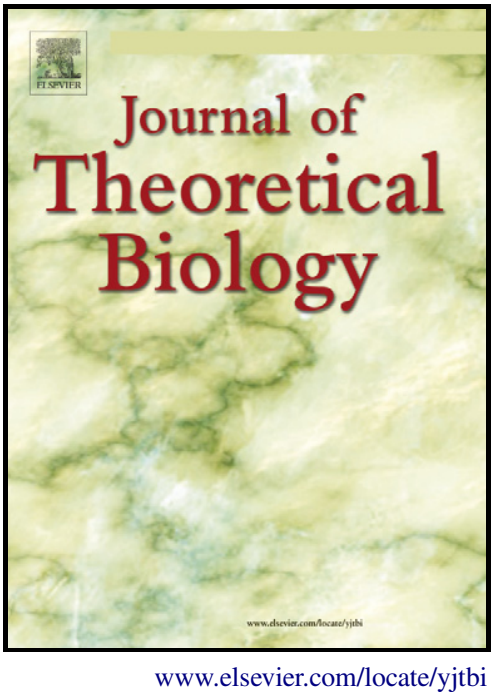

Received date: $\quad 27$ April 2010

Revised date: $\quad 2$ November 2010

Accepted date: 13 December 2010

Cite this article as: Yixian Song, Stefan Scheu and Barbara Drossel, Geographic parthenogenesis in a consumer-resource model for sexual reproduction, Journal of Theoretical Biology, doi:10.1016/j.jtbi.2010.12.020

This is a PDF file of an unedited manuscript that has been accepted for publication. As a service to our customers we are providing this early version of the manuscript. The manuscript will undergo copyediting, typesetting, and review of the resulting galley proof before it is published in its final citable form. Please note that during the production process errors may be discovered which could affect the content, and all legal disclaimers that apply to the journal pertain. 


\title{
Journal of
} Theoretical Biology

\section{Geographic parthenogenesis in a consumer-resource model for sexual reproduction}

\author{
Yixian Song ${ }^{\mathrm{a}, *, 1}$, Stefan Scheu ${ }^{\mathrm{b}}$, Barbara Drossel $^{\mathrm{a}}$ \\ ${ }^{a}$ Institute of Condensed Matter Physics, Darmstadt University of Technology, Hochschulstr. 6, D-64289 Darmstadt, \\ Germany \\ ${ }^{b}$ J.F. Blumenbach Institute of Zoology and Anthropology, \\ George August University Goettingen, \\ Berliner Str. 28, D-37073 Goettingen, Germany
}

\begin{abstract}
The phenomenon of geographic parthenogenesis is closely tied to the question of why sexual reproduction is the dominant mode of reproduction in animals and plants. Geographic parthenogenesis describes the fact that many species reproduce asexually at the boundaries of their range. We present a mathematical model that derives the dominance of sexuals at the center and the dominance of asexuals at the boundary of a species' range from exactly the same mechanism. Our model is based on a set of resources that regrow slowly and that can be consumed only by those individuals that have a suitable genotype. Genotype is implemented by a multilocus model with two alleles at each locus, and with free recombination during production of sexual offspring. The model is tailored to seasonal species with intermittent mixis and low survival of offspring, such as Daphnia and aphids. Several patches of resources are arranged in a row, with a gradient of those parameters that typically vary through the range of species. By letting sexually and asexually reproducing populations compete, we obtain the typical patterns of geographic parthenogenesis.
\end{abstract}

Key words: Asexual reproduction, Migration, Resource limitation, Spatial modeling, Structured resources

\footnotetext{
${ }^{*}$ Corresponding author

Email addresses: yixian@fkp.tu-darmstadt.de (Yixian Song), sscheu@gwdg.de (Stefan Scheu), drossel@fkp.tu-darmstadt.de (Barbara Drossel)

${ }^{1}$ Tel. 00496515 16-2914, Fax 00496151 16-3681
} 


\section{Introduction}

Any theory that aims at explaining why sexual reproduction prevails in most organisms, must be in agreement with the general patterns of parthenogenesis, in particular geographic parthenogenesis. Geographic parthenogenesis describes the fact that many species reproduce asexually at the boundaries of their range, i.e. in northern regions, at high elevations, or at the transition to deserts, while they reproduce sexually closer to the center of their range $[46,16,4,20]$. For both phenomena, sexual reproduction and geographic parthenogenesis, exist a variety of explanations and models, however, these cannot easily be linked.

Theories for sexual reproduction argue that it can purge the genome more efficiently from deleterious mutations under the appropriate conditions [36, 8, 35], and that it facilitates adaptation to a novel environment when sex leads to an increased variance in fitness [35]. A variation of the latter hypothesis, when the environment of a species is dominated by the negative influence of another species, is the Red Queen hypothesis [45, 19, 31, 33]. Furthermore, sex can restore genetic combinations that become lost due to drift but that are favored in temporally or spatially fluctuating environments [38]. In particular, environmental heterogeneity facilitates sexual reproduction, as stated in the Tangled Bank hypothesis [4], and as confirmed by a recent experimental study [3]. Natural environments are usually heterogeneous, as demonstrated by experiments that show that genetically diverse populations can better exploit resources than genetically homogeneous populations [11]. Generally, it is increasingly recognized that genetic diversity affects the performance of communities in various ways including e.g., competition for resources and community productivity [23].

Theories for geographic parthenogenesis propose that parthenogenesis is a side effect of hybridization in boundary regions [26] or of selection for polyploidy [37]. Other authors emphasize that asexuals are capable of colonizing new habitats faster $[9,30]$ or that asexual reproduction arises whenever environmental changes may have provided opportunities for shifts to asexuality [21]. In the only spatially extended mathematical model for geographic parthenogenesis that exists so far, Peck(1998) [40], showed explicitly that a sufficiently strong source-sink effect [10] can lead to a dominance of parthenogenetic reproduction in boundary regions, because sexuals cannot establish the phenotype that is optimal for this region. In this model, asexuals are assigned a lower fitness than sexuals, given the same degree of adaptation to their environment, which changes along the spatial gradient. The fitness of the sexuals in the boundary region is even lower than that of the asexuals because of the inflow of maladapted genotypes due to migration. The immigrating sexual individuals mate with the local sexual population and thus prevent it from becoming adapted to the local environment. In contrast, the local asexual genotypes can be perfectly adapted to the environment. An alternative scenario is envisaged by Gaggiotti(1994) [13] who has pointed out that geographic parthenogenesis could occur as an equilibrium phenomenon if parthenogenetic reproduction was selectively favored against sexual reproduction in the boundary regions, while sexual reproduction was favored elsewhere.

In this paper, we pursue the latter idea that the mode of reproduction prevailing at a certain location should be the one that has a selective advantage at this location. This idea has been most forcefully put forward by Bell(1982) [4] in his book "The masterpiece of nature", and it is justified by the observation that many organisms have the ability to switch between different modes of reproduction. In particular, situations of crowding and starvation elicit sexuality in organisms that reproduce asexually at other times. Because of this observation, we assume furthermore that resource availability is the main determinant of the fitness of an individual. Indeed, Glesener(1978) [16], citing the book of Ghiselin(1974) [15], pointed out that the depletion 
60 of resources favors those individuals that switch to a currently underutilized resource. Thus, sexual reproduction should be favored in heterogeneous environments with a broad spectrum of resources that have a limited availability, while parthenogenetic reproduction should prevail where the resource limitation is not important or affects both modes of reproduction in the same 64 way, as is the case in new habitats or in habitats with high mortality or small resource richness. This is the essence of the argument put forward in Bell(1982) [4].

However, older mathematical implementations of the tangled bank hypothesis [6] that include a broad spectrum of resources, while leading to the persistence of sexual reproduction despite the twofold cost of males, result in the coexistence of both modes of reproduction and can neither tolerate a continuous creation of new asexual clones, nor do they lead to the phethe lack of empirical evidence. When interpreted as predicting a greater advantage to sexual reproduction when numbers of offspring and thus sib competition are larger, it does not agree with empirical investigations [12, 27]. Burt \& Bell(1987) [5] rejected the tangled bank hypothesis on similar grounds, as mammal species that have more offspring do not have increased rates of recombination. However, these arguments have been questioned by other authors [7, 28, 18].

A different approach to the mathematical modelling of ideas related to the tangled bank hypothesis was taken by Scheu \& Drossel(2007) [42], where the number of locally coexisting genotypes was limited and drift was assigned an important role. The model is tailored to $r$ strategists with intermittent mixis and includes explicitly the interaction of the consumers with their resources. Resources are structured, and one consumer genotype therefore can only exploit a small part of the resources. Similar to older tangled bank models, it relies on quantitative genetics with a fixed genetic variance of sexuals. Slow regrowth of resources combined with stochastic effects due to restrictions on the number of locally coexisting genotypes, leads to the prevalence of sexual reproduction in spite of the cost of producing males. Interestingly, in this model the advantage of sexual reproduction does not increase with increasing density, since this would decrease the stochastic effects due to different resources being exploited at different times. The mentioned results hold for a wide range of parameters [42, 1]. However, with increasing mortality or decreasing resource richness, the dominant mode of reproduction becomes asexual. This finding suggests that the model can also be used to generate a scenario that shows geographic parthenogenesis.

In the following, we will investigate a modified model that differs in two respects from the one by Scheu \& Drossel(2007) [42]. First, instead of using quantitative genetics, the modified model is genetically explicit and uses several diallelic loci with free recombination. This feature permits to study the effect of recombination on the genetic diversity of the population, which was not possible in the quantitative genetic model. Second, the modified model includes a spatial dimension, in order to generate a setup that can show geographic parthenogenesis. By arranging patches of resources along a habitat that stretches from regions of low consumer mortality to regions of high consumer mortality or from regions of large resource richness to regions of small resource richness, we will find a pattern resembling the phenomenon of geographic parthenoasexuals at the boundary of a species' range can be caused by exactly the same mechanism.

In the next section, we will define the rules of the model in more detail. In the following two sections, we will present the results of computer simulations and explain how they follow from the rules of the model. We conclude this paper by placing the results in a wider context. 


\section{The Model}

Twenty resource patches are arranged along a one-dimensional chain. A gradient of up to three patch features (specified below) reflects the change in habitat properties from the center (patch 1) to the boundary (patch 20) of a species' range. Each patch contains up to $L=256$ different "resources", which are labeled by binary numbers from 0 to 255 , and which we will also name "niches" in the following. Consumers are characterized by their mode of reproduction and by their genotype, which for simplicity is denoted by the same numbers as the resources. The genotype of the consumers is represented by a set of 8 diallelic loci. When the two alleles at each locus are labelled 0 and 1 , the genotype can be represented by a binary number with 8 bits. A consumer is best adapted to the resource the label of which matches its genotype. This method of implementing a genotype and matching it with resources is similar to the one chosen by Gavrilets (2005) [14]. The resources in this model do not necessarily represent different species, but the different possible ways in which consumers can specialize on resources that differ in some respect. The eight degrees of freedom can thus represent different location (humid, less humid, more or less shady), different manifestation (producing certain defenses or not, having a harder surface), or different parts (upper or lower leaves) and so on of the same type of resource. Due to different search patterns, different digestive abilities, different morphological features, etc., different individuals exploit resources in different ways. Indeed, experiments show that genetically diverse populations can better exploit resources than genetically homogeneous populations [11]. Svanbäck and Bolnick (2005) [44] argue that individuals that differ in their ability to find, handle or digest alternative resources may have different diets.

The model is initiated with a maximum resource biomass $R_{j}=R_{\max }=100$ for each resource, and with some niches being occupied by sexual or asexual individuals. The life cycle of the consumers is tailored to that of species with intermittent mixes such as Daphnia [4, 20]. Thus, the biomass $P_{i}$ of consumer number $i$, which is best adapted to the niche $i$ increases from the initial value $P_{i}=1$ by parthenogenetic reproduction during the season according to the equation

$$
\dot{P}_{i}=\lambda \sum_{j=1}^{L} \alpha_{i j} P_{i} R_{j}-d P_{i},
$$

while the resource biomass decreases according to

$$
\dot{R}_{j}=-\sum_{i} \alpha_{i j} P_{i} R_{j}
$$

where the sum is taken over all consumers in the same patch. The ecological efficiency $\lambda$ was chosen to be 0.2 [43]. The coupling strength $\alpha_{i j}$ between consumer and resource was set equal to 1 for $i=j$ and equal $e^{-k}$ (with $k$ being $0,0.5$ or 1 ) when $i$ and $j$ differ in one bit. Otherwise it was set to zero. In most of the simulations reported below, we used $k=0$, which means that each consumer uses with equal efficiency all 9 resources available to it.

In order to mimic increasing harshness of the environment as the patch index increases from center to the boundary of a species' range, we let the death rate $d$ increase linearly with increasing patch index, or we let the number of resources $L$ decrease linearly. Variation in the number of resources was implemented such that the resources with the largest labels were removed.

Population dynamics (1) is applied for each consumer until $\dot{P}_{i}$ becomes zero, when the consumer stops feeding and growing. After all consumers have stopped growing, the season is ended. 
We assume that sexual individuals mate at random within the patch, where there is an equal number of males and females, and all females lay the same large number of eggs per biomass. Thus the number of eggs per parent biomass laid by asexuals is twice as large as that of sexuals. The precise number of eggs per female is irrelevant for the following, since the number of eggs that give rise to a surviving individual is small and independent of the total egg number. Next, the starting configuration for the next season is calculated: Resources regrow partially according to the equation

$$
\Delta R_{j}=G\left(1-R_{j} / R_{\max }\right)=\frac{G}{R_{\max }} \underbrace{\left(R_{\max }-R_{j}\right)}_{R_{j}^{\text {consumed }}},
$$

with the regrowth percentage $G \leq R_{\max }$, which can vary between patches. The number of consumer individuals in each patch at the beginning of a new season is chosen from a Poisson distribution with mean value $n$. Each egg is chosen with the same probability to give rise to such a surviving individual. Therefore, the mode of reproduction of each individual at the beginning of the new season is sexual with probability

$$
p_{\mathrm{s}}=\frac{\sum_{i \text { sex. }} P_{i}}{\sum_{i \text { sex. }} P_{i}+2 \sum_{i \text { parthenog. }} P_{i}},
$$

and parthenogenetic with probability $1-p_{\mathrm{s}}$, with the $P_{i}$ being the biomasses at the end of the previous season. The factor 2 in the denominator takes into account the twofold cost of sex, due to half of the sexual individuals being male. The genotype of a parthenogenetic individual is randomly chosen among the genotypes of parthenogens present at the end of the previous season, with weights proportional to their final biomass, and with each bit of the genotype being mutated with a small probability $\mu$. The genotype of a sexual individual is determined by randomly choosing two sexual parent genotypes with probabilities proportional to their biomass at the end of the previous season, and by generating the offspring by free recombination at every locus. Just as for asexual offspring, each bit of the genotype is then mutated with a small probability $\mu$. Finally, with a small probability $u=0.001$, the offspring of sexual individuals becomes parthenogenetic, implementing the 'frozen niche variation hypothesis' of Vrijenhoek(1979) [47], which is based on the fact that parthenogenetic lineages can arise in sexual populations.

Before onset of resource consumption, each individual is allowed to migrate to each neighboring patch with a migration probability $\eta$. Alternatively, we also implemented the case of egg dispersal, where "migration" occurs before selection of the surviving eggs.

We iterated this process for 200 seasons and averaged the resulting final proportion of sexuals in each patch over 1000 runs of the computer simulation.

\section{Results}

Fig. 1 shows the average proportion of sexuals in each patch that was obtained after 200 seasons, when an equilibrium had been reached. In the first graph (Fig. 1A), the death rate $d$ was increased with increasing patch index, varying in total by a factor of 3.5 ; in the second graph (Fig. 1B) the number of different resources, $L$, was decreased from 256 to 66 with increasing patch index, in the third (Fig. 1C) and fourth graph (Fig. 1D) the percentage $G$ of consumed resource biomass that regrows between subsequent seasons was decreased from 40 to 11.5 , together with a change in $d$ or $L$. In each of the four cases, the proportion of sexuals decreases 
from (almost) $100 \%$ to (almost) $0 \%$ with increasing patch index, i.e., with increasing harshness of the environment. The model thus reproduces the phenomenon of geographic parthenogenesis.

These results are independent of the initial distribution of genotypes and modes of reproduction. We obtained the same graphs when we tested the following four initial situations: (a) 25 asexual and 25 sexual genotypes in random niches in each patch, (b) 50 sexual genotypes and no asexuals in random niches in each patch, (c) 25 sexual and 25 asexual genotypes in random niches in the first patch, with all other patches being empty, (d) 50 sexual genotypes in random niches in the first patch, with all other patches being empty. The first case imposes no bias towards one mode of reproduction, the second case shows the long-term effect of the rare production of asexual clones within sexual populations, the third and fourth case simulate the colonization of a new habitat by a population that initially displays either both modes of reproduction or reproduces only sexually.

The pattern of geographic parthenogenesis is robust against changes in other ecological factors, which are investigated in the following.

Fig. 2 shows the influence of the migration rate $\eta$ and of the mutation rate per locus $\mu$. The advantage of sexuals increases at first with increasing migration rate (Fig. $2 \mathrm{~A}$ ), because new genetic variance is brought in from neighboring patches, thus preventing a decline of genetic variance of the local sexual populations. When the migration rate becomes even larger, the proportion of sexuals decreases more and more, because the asexual genotypes can be present in several patches at the same time, which decreases their extinction rate and enables them to benefit from the twofold cost per season incurred by the sexuals. We also investigated the case where migration happens before determining the genotypes of the individuals at the beginning of a new season (which can be interpreted as egg dispersal). The curves changed only little with this modification. In Fig. $2 \mathrm{~B}, \mu$ is increased from the first to the last patch reflecting the fact that stressful environment can elicit a high mutation rate $[17,2]$. In this case, the proportion of sexuals decreases from (almost) $100 \%$ to (almost) $0 \%$ with increasing patch index, i.e., with increasing environmental stress.

A decrease of the regrowth percentage $G$ without a simultaneous increase in the death rate or decrease in the number of the different resources, leads to an increase in the percentage of sexuals instead of a decrease. A large value of $G$ favors parthenogenetic reproduction, since there is no advantage of exploring new niches when resources grow very fast. A change of $n$ (either between patches or overall) does not change qualitatively our results, as long as $n$ is considerably smaller than $L$ even at the center, i.e., as long as there are enough unexploited niches that can be used by sexual offspring, and as long as $n$ is large enough to support a significant genetic variance.

We also investigated the effect of a different coupling strength to neighboring resources. For $k=0.5$ or 1 , the parameter region where sexual reproduction dominates becomes different (because a larger $k$ has a similar effect to a larger $d$ (Eq. 1)), but geographic parthenogenesis is also observed. When the number of resources on which a genotype can feed is extended to a larger neighborhood, there is no longer any advantage to sexual reproduction, because there are no unexploited resources left even for smaller values of $n$. We also varied the number of loci. When we performed our simulations with 9 loci and up to $L=512$ different resources, the parameter interval where sexual reproduction dominates became larger. With 5 or less loci, we could not obtain a proportion of sexuals close to 1, because the advantage to sexuals of having a larger genetic variance is not strong enough. With 7 loci, we could obtain results similar to those with 8 loci only with sufficiently large $k$, i.e. when an individual could not feed on neighboring resources. With smaller $k$, each genotype would be able to exploit 8 resources, which is roughly $1 / 11$ of all resources. In order to have unused resources that could be exploited by the sexual 
offspring, the population size would have to be very small - and then the genetic variance would be lost so quickly that sexuals have no advantage. With 6 loci, the parameter range for which we found a dominance of sexual reproduction was much smaller.

\section{4. Analysis}

In order to understand the simulation results in greater depth, let us first consider the case of a single patch (or, equivalently, a set of isolated patches). Due to the twofold cost of producing males incurred once per season, sexuals can only have an advantage when there is sufficient ge-

214 netic variance in the patch so that offspring can be generated that are capable of exploiting unused resources. We therefore evaluate in the following the genetic variance of sexuals and asexuals

216 under different conditions. If there was no selection, the genetic variance would be independent at each locus and fully determined by drift. With a population size of $n$ and an initial frequency of 50 percent of each of the two alleles, the probability of not having lost one of the two alleles after $g$ seasons can be estimated to be roughly of the order $e^{-g / n}$ (since the number of individuals

220 with a given allele changes per generation by the order of $\sqrt{n} / 2$, and since the number of random steps of this size required to cover the distance $n / 2$ is of the order of $n$ ). One can therefore expect 222 the number of loci with two alleles to become very small within 100 seasons. This is indeed the case, as shown in Fig. 3. Without mutations, the genetic variance eventually declines to zero

224 (Fig. 3A). We performed the simulation once for a purely sexual population and once for a purely asexual population, without taking into account selection, i.e., with each individual having the same chance of being the parent of a child in the next season. While the number of diallelic loci behaves exactly in the same way in both cases, the number of genotypes is considerably larger for the sexuals, due to recombination. When the mutation rate is nonzero (Fig. 3B), the genetic variance reaches a nonzero stationary value. As long as the mutation rate is small, the sexuals

230 have a clear advantage over the asexuals in terms of genetic variance. A single mutation in a sexual individual at a locus that has lost one of the two alleles can generate many new genotypes 232 through recombination with all the other genotypes. In contrast, a mutation in an asexual individual generates only one new genotype. If the mutation rate is higher, this advantage of the sexuals

234 becomes smaller. Fig. 3B shows the result obtained with $\mu=0.016$, where the number of sexual genotypes is less than twice the number of asexual genotypes, which means that the two-fold 236 cost of sex cannot be compensated (remember that this simulation was performed without taking into account differences in resource availability). Therefore, the patches with high mutation rate $\mu$ are dominated by parthenogenetic reproduction (Fig. 2B). When resource dynamics are taken into account, rare alleles will in fact have a considerable advantage in sexual species due to being

240 able of exploiting unused resources. The loss of an allele becomes therefore less likely. This is shown in Fig. 4A and Fig. 4B for a zero and a nonzero mutation rate, respectively. A comparison 242 with Fig. 3 demonstrates a large difference between sexuals and asexuals: Even without mutation, the sexuals usually lose none of their alleles, because rare alleles can recombine with other

244 genotypes to exploit underutilized resources. Mutation does not confer an additional advantage. In contrast, the number of asexual genotypes depends strongly on the mutation rate. Neverthe-

246 less, just as for the sexuals, resource dynamics prevents the loss of rare alleles. The reason is that asexual lineages that had plenty of food in the previous season and lay many eggs, produce off248 spring that feed all on the same (limited) resource in the present season. At the end of the season, the number of individuals of this genotype will be approximately the same as when there initially

250 had only been one individual, meaning that additional individuals of the same genotype at the beginning of the season do not lead to a larger number of individuals at the end of the season. The 
252 total number of asexual genotypes decreases in the absence of mutations to a single-digit number because genotypes with overlapping resources have a disadvantage compared to those without resource overlap, and the number of different genotypes declines until the remaining individuals have (almost) no overlap.

Finally, we evaluated the number of loci $N_{\mathrm{di}}$ for which both alleles are present in the population, and the number of different genotypes $N_{\text {ge }}$ for the full model, for the simulation parameters of Fig. 2A. The results are shown in Fig. 5. One can see that the sexuals quickly lose their genetic variance in patch number 20, where death rate is high, and where asexuals have the advantage of having more offspring. On the other hand, the sexuals can maintain their genetic variance in the first patch, where they succeed at producing more offspring than the asexuals, due to being capa-

262 ble of utilizing more resources. In both cases, the winning mode of reproduction can already be identified during the first few season, due to the rapid decline of the other mode of reproduction. This means that in the central patches sexual reproduction is not only a long-term advantage, but also a short-term advantage.

266 One can also see that migration can further increase the genetic variance of sexuals as long as the migration rate is not too large. Interestingly, over the entire set of patches, almost all alleles are present irrespective of the mode of reproduction $\left(N_{\mathrm{di}}\right.$ in Fig. $\left.5 \mathrm{C}\right)$. This proves once again that structured resources prevent the decline of genetic variance in general, by preferring rare alleles.

\section{Conclusions}

In this paper, we have modified and extended a mathematical model for sexual reproduction that is based on a broad spectrum of limited resources that regrow slowly [42], in order to obtain the phenomenon of geographic parthenogenesis. In this model, the number of genotypes that can coexist at the same place is limited, thus providing an advantage to sexuals whenever the offspring can acquire a genotype that enables it to exploit unused resources. In contrast to the original model [42], which was based on quantitative genetics, we now used a genetically explicit model. This permitted us to generate sexual offspring via recombination, and to evaluate explicitly the number of different alleles present in the populations. The two different implementations used in the two models, represent two limiting cases: The quantitative genetic model

280 includes only two parameters to specify a resource and a genotype, both of which are continuous and Gaussian distributed with a constant genetic variance, which implies that they result from the superposition of the effects of many genes that contribute additively to it. The genetically explicit model uses eight parameters to specify a resource and a genotype, with each parameter taking only two values. Both genetic implementations lead to a prevalence of sexual reproduction when there is a sufficient amount of unexploited resources and when the ability to exploit these resources leads to an increase in the number of offspring. This demonstrates that the outcome of the model is sufficiently generic and does not rely on the precise way in which genetics is implemented. If only the following three requirements are met, sexual reproduction can prevail over a wide parameter range: (i) the number of locally coexisting genotypes is so small that they can use only part of the resources with good efficiency. (ii) Sexual parents can have offspring that can efficiently use resources that their parents could not efficiently use. (iii) Resources regrow slowly.

One can imagine various other variants of the model that satisfy these requirements when parameter values are chosen in an appropriate range. For instance, we performed simulations of a genetically explicit version of the quantitative genetic model, where each trait value resulted from the addition of the values at 20 diallelic loci. This version of the model displays similar 
results when there is a sufficiently large number of patches that are coupled by migration, so that alleles do not get lost too quickly. We also performed simulations with a fourth version of the model: Instead of letting each individual feed on the resource(s) it is best adapted to, we placed each individual directly into a niche, where it could feed with an efficiency that depends on the extent of agreement between the trait value of the individual and the niche value. This version 302 of the model permits using much larger population sizes, since only part of the individuals feed with maximum efficiency; again it displays the prevalence of sexual reproduction.

In the present paper, we have extended our previous model [42] such that it is composed of several patches, which are arranged as a one-dimensional chain extending from the center to the boundary of a species' range, with migration between neighboring patches, and with a spatial gradient of those parameters that may vary as the environment becomes harsher towards the boundary. Parameters were chosen such that sexual reproduction would prevail at the center and that asexual reproduction would prevail at the boundary if there was no migration. Our simulations have shown that the spatially extended system with migration reaches an equilibrium distribution of sexual and asexual individuals such that no asexuals are at the center and no

312 sexuals are at the boundary, as long as the migration is not too large. While we have demonstrated this pattern of geographic parthenogenesis by using the genetically explicit model, it occurs also for the quantitative genetic model and the other modifications mentioned in the previous paragraph, when patches are arranged in a similar way. We have thus found a class of models

316 that can produce the phenomenon of sexual reproduction and the phenomenon of geographic parthenogenesis by exactly the same mechanisms.

The finding that mortality has a strong influence on the reproductive mode is in agreement with the observation that asexual organisms are more frequent in transient, ecotonal, disturbed or marginal environments, like deserts [25], which are associated with high mortality.

We have explained the results of the computer simulations by considering the genetic variance of the sexuals as function of the parameters. Whenever recombination, mutation and migration together allow for many more sexual than asexual genotypes, sexual reproduction wins over parthenogenetic reproduction when resources are scarce, despite the twofold cost of males that sexuals incur once per season.

The model used in this paper, of course, is only a simplified description of sexual reproduction in a spatially inhomogeneous environment. In the simulations, we used 20 patches with up to 256 different resources each, and the consumers were represented by a genotype of 8 bits and an average population size of 50, which can barely capture the extent and importance of overall spatial variation and local restriction of resources and consumer genotypes. Nevertheless, the model demonstrates nicely the advantage of sexual reproduction wherever it enables consumers 332 to exploit the resources more efficiently, and the transition to parthenogenesis wherever there is no advantage of consumers switching to other resources.

334 Other theories for the evolution of sex cannot easily be extended to generate geographic parthenogenesis. Contrary to geographic parthenogenesis, lottery models [48] predict that sexual

336 reproduction is favored at the boundary of a species' range, where the habitat is capricious and unpredictable. A spatially extended model by Salathe(2006) [41], which is based on Muller's 338 ratchet decreasing the fitness of asexuals while they invade a sexual population, predicts the dominance of asexuals in marginal habitats, with small populations that can be invaded rapidly.

340 However, such an asexual invader could persist only temporarily, until its fitness has decreased to the point where it dies out or a sexual invader replaces it again. Geographic parthenogenesis

342 therefore cannot be an equilibrium phenomenon in that model. Models based on the Red Queen mechanism $[24,19]$ require a decreasing impact of parasites on their host towards the boundary 
344 of the host's range in order to produce geographic parthenogenesis. Such models require assumptions about how host and parasite change with the geographic location [32]. Indeed, hosts are more likely to be exposed to a larger number of parasites in the center of their range which weakens the Red Queen mechanism [29] and therefore the advantage of sexual reproduction.

348 Further, Howard(1994) [22] demonstrated that a model that only includes the effect of parasites reducing the fitness of the host, is not sufficient to explain the maintenance of sex. Finally, many mathematical models on the maintenance of sexual reproduction are based on population genetics [34, 39], where fitness values are assigned to genotypes. Since such models do not consider explicitly the ecological environment, there is no straightforward way to extend them to explain geographic parthenogenesis. Overall, processes such as Muller's ratchet, interaction with exites, and interactions between genes, may all affect the mode of reproduction. However, resources are fundamental and universal, no living organism can survive or even reproduce without resources. Our model including a broad spectrum of limited resources, together with stochastic effects (drift) is unique in providing a generally satisfying explanation for the phenomenon of geographic parthenogenesis and for the general dominance of sexual reproduction.

Our model is limited by the fact that it is based on equilibrium dynamics and therefore does not take into account the ever continuing change of the biotic and abiotic environment. The set of resources used in our model is the same in every patch and over the duration of the simulation.

362 Recent work on the advantages of sexual reproduction [35, 39] focuses more on the ability of sexually reproducing organisms to adapt to new challenges. A combination of these approaches

364 with an explicit modelling of structured resources could be an important step towards a synthesis of the different theories of evolution and maintenance of sex.

366 Acknowledgment This study was funded by the Deutsche Forschungsgemeinschaft (DFG) under contract number Dr300/6.

\section{References}

[1] Ament, I., Scheu, S. and Drossel, B. 2008. Influence of spatial structure on the maintenance of sexual reproduction. J. Theor. Biol. 254:520-528.

[2] Baer, C. F., Miyamoto, M. M. and Denver, D. R. 2007. Mutation rate variation in multicellular eukaryotes: causes and consequences. Nat. Rev. Genet. 8:619-631.

[3] Becks, L., Agrawal, A. F. 2010. Higher rates of sex evolve in spatially heterogeneous environments. Nature doi:10.1038/nature09449.

[4] Bell, G. 1982. The masterpiece of nature: the evolution and genetics of sexuality. Croom Helm, London.

376 [5] Burt, A. and Bell, G. 1987. Mammalian chiasma frequencies as a test of 2 theories of recombination. Nature 326:803-805.

[6] Case, T. J., Taper, M. L. 1986. On the coexistence and coevolution of asexual and sexual competitors. Evolution 40:366-387.

380 [7] Charlesworth, B. 1987. Red queen versus tangled bank models. Nature 330:116-117.

[8] Charlesworth, D. and Charlesworth, B. 1987. Inbreeding depression and its evolutionary consequences. Ann. Rev. Ecol. Syst. 18:237-268.

[9] Cuellar, O. 1977. Animal parthenogenesis. Science 197:837-843.

384 [10] Dias, P. C. 1996. Sources and sinks in population biology. Trends Ecol. Evol. 11:326-330.

[11] Doncaster, C. P., Pound, G. E. and Cox, S. J. 2000. Nature 404:281-285.

386 [12] Ellstrand, N. C. and Antonovics, J. 1985. Experimental studies of the evolutionary significance of sexual reproduction .2. a test of the density-dependent selection hypothesis. Evolution 39:657-666.

388 [13] Gaggiotti, O. E. 1994. An ecological model for the maintenance of sex and geographic parthenogenesis. J. Theor. Biol. 167:201-221.

390 [14] Gavrilets, S. and Vose, A. 2005. Dynamic patterns of adaptive radiation. P. Natl. Acad. Sci. USA 102:18040-18045. [15] Ghiselin, M. T. 1974. The economy of nature and the evolution of sex. University of California Press, Berkeley. 
392 [16] Glesener, R. R. and Tilman, D. 1978. Sexuality and the components of environmental uncertainty: clues from geographic parthenogenesis in terrestrial animals. Am. Nat. 112:659-673.

394 [17] Goho, S. and Bell, G. 2000. Mild environmental stress elicits mutations affecting fitness in Chlamydomonas. $P$. R. Soc. Lond. B. Bio.267:123-129.

[18] Greenwood, J. J. D. 1987. Red queen versus tangled bank models. Nature 330:117-117.

[19] Hamilton, W. D. 1980. Sex versus non-sex versus parasite. Oikos 35:282-290.

398 [20] Hebert, P., Ward, R. D. and Weider, L. J. 1988. Clonal-diversity patterns and breeding-system variation in Daphnia pulex, an asexual-sexual complex. Evolution 42:147-159.

400 [21] Hörandl, E. 2009. A combinational theory for maintenance of sex. Heredeity 103:445-457.

[22] Howard, R. S. and Lively, C. M. 1994. Parasitism, mutation accumulation and the maintenance of sex. Nature 367:554-557.

[23] Hughes, A. R., Inouye, B. D., Johnson, M. T. J., Underwood, N., Vellend, M. 2008. Ecological consequences of genetic diversity. Ecology Letters 11:609-623.

[24] Jaenike, J. 1978. A hypothesis to account for the maintenance of sex within populations. Evol. Theor. 3:191-194.

406 [25] Kearney, M. R. 2003. Why is sex so unpopular in the Australian desert? Trends Ecol. Evol. 18:605-607.

[26] Kearney, M. 2005. Hybridization, glaciation and geographical parthenogenesis. Trends Ecol. Evol. 20:495-502.

408 [27] Kelley, S. E. 1989. Experimental studies of the evolutionary significance of sexual reproduction .6. a greenhouse test of the sib-competition hypotheses. Evolution 43:1066-1074.

410 [28] Koella, J. C. 1987. Red queen versus tangled bank models. Nature 330:117-118.

[29] Kouyos, R. D., Salathe, M., Otto, S. P. and Bonhoeffer, S. 2009. The role of epistasis on the evolution of recombination in host-parasite coevolution. Theor. Popul. Biol. 75:1-13.

[30] Law, J. H. and Crespi, B. J. 2002. The evolution of geographic parthenogenesis in Timema walking-sticks. Mol. Ecol. 11:1471-1489.

[31] Lively, C. M., Dybdahl, M. F. 2000. Parasite adaptation to locally common host genotypes. Nature 405:679-681.

[32] Lively, C. M. 2001. Trematode infection and the distribution and dynamics of parthenogenetic snail populations. Parasitology 123:S19-S26.

418 [33] Lively, C. M. 2009. The maintenance of sex: host-parasite coevolution with density-dependent virulence. J. Evol. Biol. 22:2086-2093.

420 [34] Martin, G., Otto, S. P. and Lenormand, T. 2006. Selection for recombination in structured populations. Genetics 172:593-609.

[35] Morran, L. T., Parmenter, M. D. and Phillips, P. C. 2009. Mutation load and rapid adaptation favour outcrossing over self-fertilization. Nature 462:350-352.

[36] Muller H. J. 1932. Some genetic aspects of sex. Am. Nat. 66:118-138.

[37] Otto, S. P. and Whitton, J. 2000. Polyploid incidence and evolution. Annu. Rev. Genet. 34:401-437.

426 [38] Otto, S. P. and Lenormand, T. 2002. Resolving the paradox of sex and recombination. Nat. Rev. Genet. 3:252-261.

[39] Otto, S. P. 2009. The evolutionary enigma of sex. Am. Nat. 174:S1-S14.

[40] Peck, J. R., Yearsley, J. M. and Waxman, D. 1998. Explaining the geographic distributions of sexual and asexual populations. Nature 391:889-892.

430 [41] Salathé, M., Salathé, R., Schmid-Hempel, P. and Bonhoeffer, S. 2006. Mutation accumulation in space and maintenance of sexual reproduction. Ecol. Lett. 9:941-946.

432 [42] Scheu, S. and Drossel, B. 2007. Sexual reproduction prevails in a world of structured resources in short supply. Proc. Roy. Soc. B 274:1225-1231.

434 [43] Stephens, D. W. and Krebs, J. R. 1986. Foraging Theory. Princeton University Press, Princeton, NJ.

[44] Svanbäck, R. and Bolnick, D. I. 2005. Intraspecific competition affects the strength of individual specialization: an optimal diet theory method. Evol. Ecol. Res. 7:993-1012.

[45] Van Valen, L. 1973. A new evolutionary law. Evol. Theor. 1:1-30.

[46] Vandel, A. 1928. La parthénogénèse géographique: contribution à l'étude biologique et cytologique de la parthénogénèse naturelle. Bull. Biol. Fr. Belg. 62:164-281.

440 [47] Vrijenhoek, R. C. 1979. Factors affecting clonal diversity and coexistence. Am. Zool. 19:787-797.

[48] Williams, G. C. 1966. Adaptation and natural selection. Princeton University Press, Princeton, NJ. 

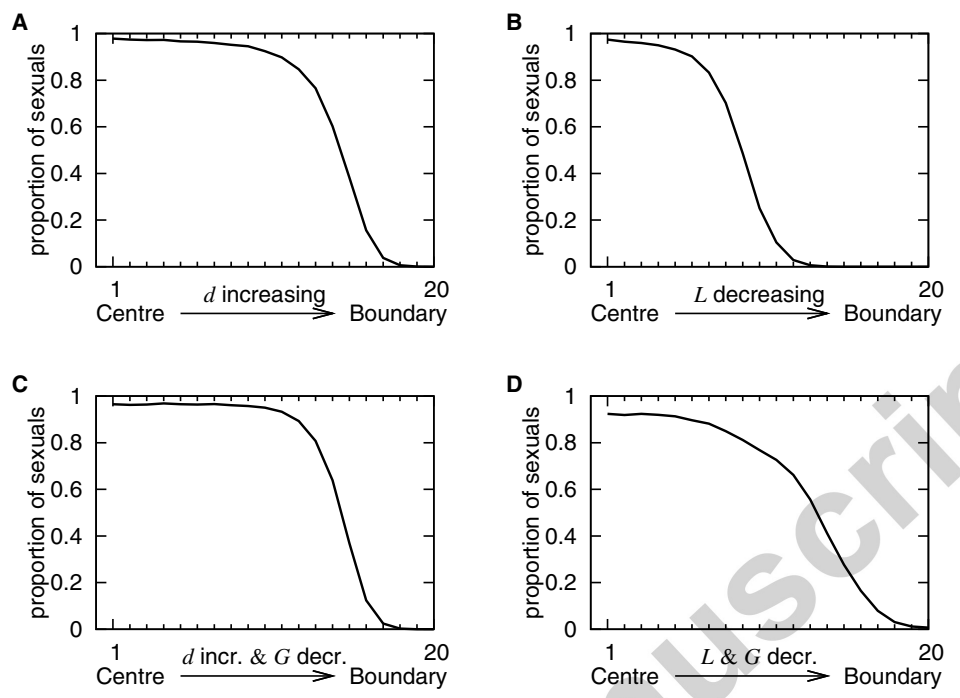

Figure 1: Average equilibrium proportion of sexuals in the 20 patches with the patch index increasing from the center (1) to the boundary (20). The parameters that vary between patches are (A) the consumer death rate $d$, (B) the number of different resources $L,(\mathrm{C})$ the death rate $d$ and the regrowth percentage of resources $G$, (D) $G$ and $L$. The death rate $d$ is increased in constant steps from 30 to 106 , the number of different resources $L$ is decreased from 256 to 66 in (B) and to 142 in (D), and the regrowth percentage of resources $G$ is decreased from 40 to 11.5 in (C) and (D). The parameters that are not varied between patches are $d=30, L=256, G=20$. The remaining parameters are: average number of initial individuals per patch $n=50$, mutation rate per locus $\mu=0.002$, sexual-to-asexual mutation rate $u=0.001$, migration rate $\eta=0.01$, and equal coupling strength to all 9 resources available to a genotype (i.e., $k=0$ ) .
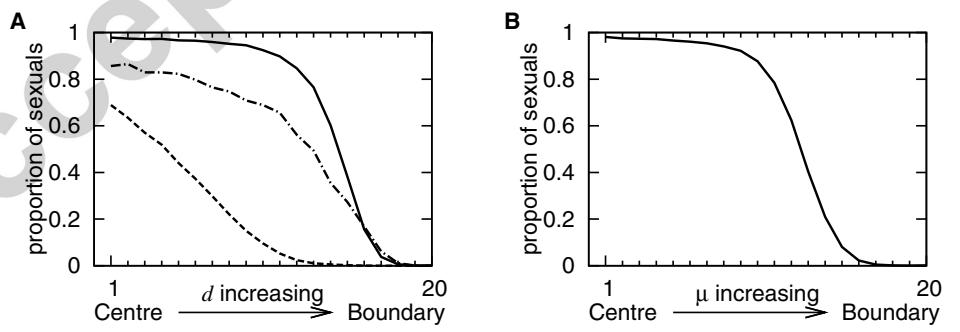

Figure 2: Average equilibrium proportion of sexuals in the 20 patches with the patch index increasing from the center (1) to the boundary (20). The parameters that vary between patches are (A) the consumer death rate $d$ (in constant steps from 30 to 106) for three different values of the migration rate $\eta=0$ (dot-dashed), $\eta=0.01$ (solid), $\eta=0.1$ (dashed), (B) the mutation rate per locus $\mu$ (increasing from 0 to 0.019 ). The parameters that are not varied between patches are $d=30, \mu=0.002, L=256, G=20, n=50, u=0.001, k=0$. 

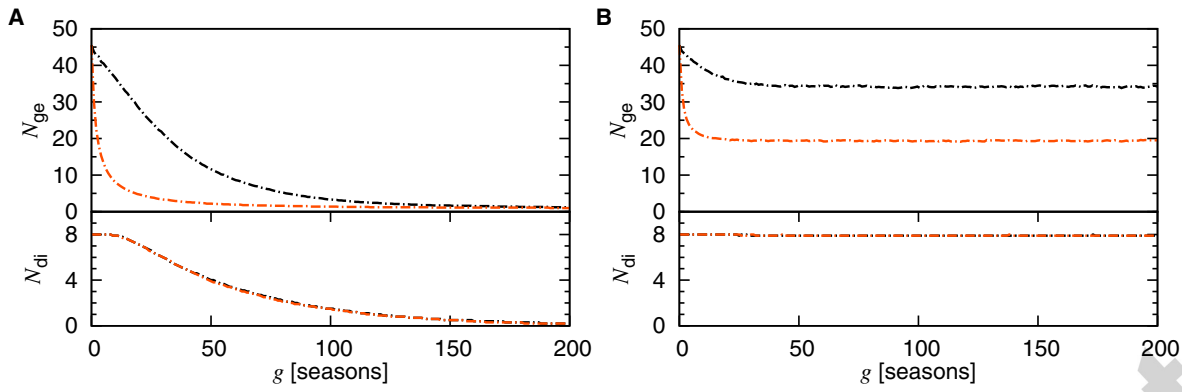

Figure 3: Average number of diallelic loci $N_{\mathrm{di}}$ and genotypes $N_{\mathrm{ge}}$ for a sexual (black lines) or parthenogenetic population (red lines) of average size $n=50$ at the beginning of each season as function of time. The graphs are evaluated for a null-model, i.e., only with drift and without selection, and the two reproductive modes are considered independently from each other. The mutation rate per locus is (A) $\mu=0$ and (B) $\mu=0.016$. The average number of diallelic loci evolves in the same way for both sexual and parthenogenetic reproduction.
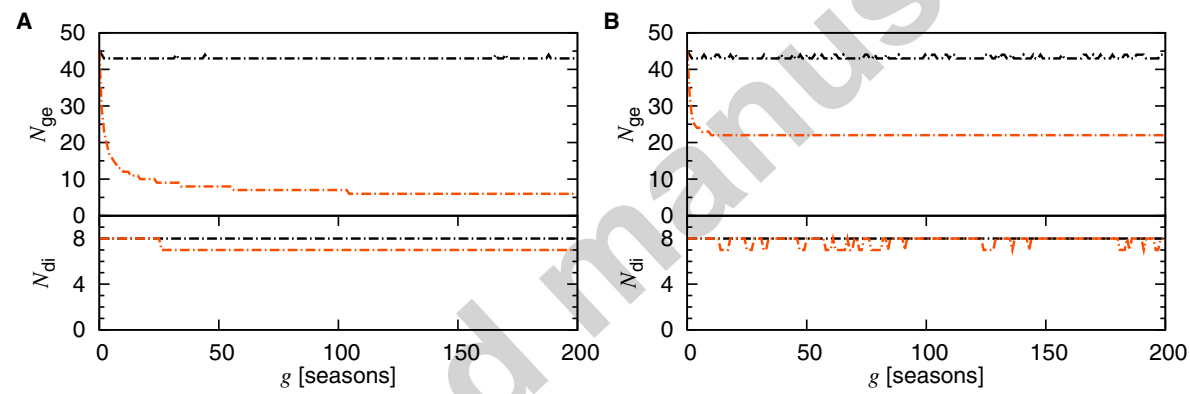

Figure 4: Same as Fig. 3, but with resource dynamics. The additional parameters are: death rate $d=30$, number of different resources $L=256$, regrowth percentage of resources $G=20$, average number of initial individuals per patch $n=50$, and equal coupling strength to all 9 resources available to a genotype (i.e., $k=0$ ).
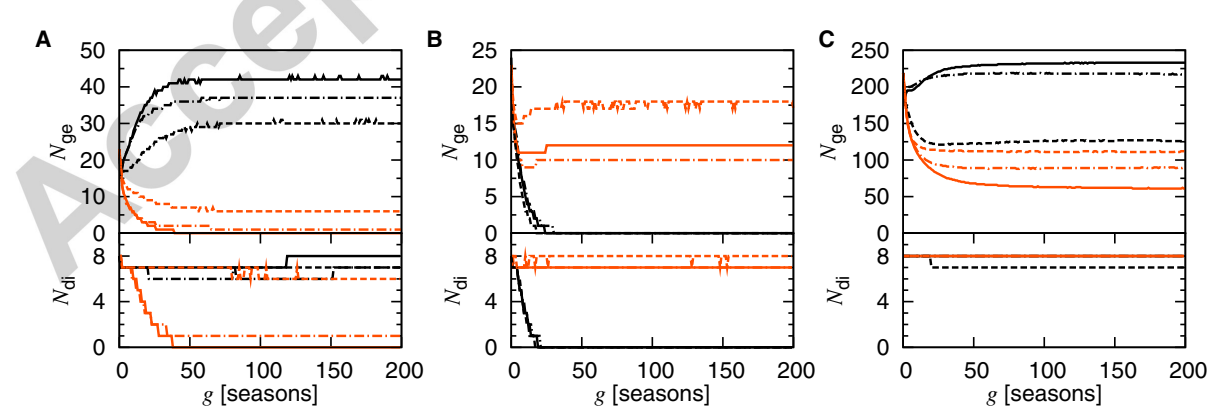

Figure 5: Average number of loci $N_{\mathrm{di}}$ with two alleles and of different genotypes $N_{\mathrm{ge}}$ for a situation where sexual (black lines) and parthenogenetic reproduction (red lines) compete with each other, as function of time. The simulations were performed for the parameter set of Fig. 2A. (A) first patch $m=1$, (B) $m=20$. (C) all 20 patches. Dot-dash line: $\eta=0$; solid lines: $\eta=0.01$; dash line: $\eta=0.1$. 